Article

\title{
An Orthogonal Model to Study the Effect of Electrospraying Parameters on the Morphology of poly (d,1)-lactide-co-glycolide (PLGA) Particles
}

\author{
Ming-Yi Hsu ${ }^{1,2}$, Chu-Han Feng ${ }^{2}$, Yen-Wei Liu ${ }^{2}$ and Shih-Jung Liu ${ }^{2,3, * \mathbb{D}}$ \\ 1 Department of Medical Imaging and Intervention, Chang Gung Memorial Hospital at Linkou, \\ Tao-Yuan 33302, Taiwan; m7259@cgmh.org.tw \\ 2 Department of Mechanical Engineering, Chang Gung University, Tao-Yuan 33302, Taiwan; \\ tabu11440@gmail.com (C.-H.F.); anglevvings@gmail.com (Y.-W.L.) \\ 3 Department of Orthopedic Surgery, Chang Gung Memorial Hospital-Linkou, Tao-Yuan 33305, Taiwan \\ * Correspondence: profsjliu5347@gmail.com
}

Received: 19 February 2019; Accepted: 12 March 2019; Published: 14 March 2019

\begin{abstract}
Electrospraying has been recognized as an important technique for the production of microparticles for pharmaceutical applications. Nevertheless, the produce of poly $(\mathrm{d}, \mathrm{l})$-lactide-co-glycolide (PLGA) microspheres with a desired size distribution remains a challenge. We conducted an empirical study, based on a fractional orthogonal design, to optimize the size distribution of electrosprayed particles. The influence of different processing factors, including PLGA concentration in the solution, volumetric flow rate of the solution, travel distance between the needle tip and the collection, voltage applied to the polymer solution, size of the needle, and type of solvent on the electrospraying of polymeric microparticles was investigated. After electrospraying, the size distribution of the PLGA particles was characterized and analyzed. Among the selected factors, the type of solvent used was the principal factor affecting the particle size of electrosprayed microspheres. Hexafluoro-2-propanol (HFIP) electrosprayed microparticles with the smallest diameter. However, hollowed particles could be seen among these microspheres. Dichloromethane (DCM) was found to electrospray microspheres with a fairly spherical geometry, while trichloromethane (TCM) electrosprayed particles with relatively rougher surfaces. Finally, the particle size of sprayed microspheres decreases somewhat with the polymer concentration and travel distance.
\end{abstract}

Keywords: electrospraying; processing parameters; microparticles

\section{Introduction}

Electrospraying, or known as electrostatic hydrodynamic atomization process, is able to produce tiny particles with micro- and nano- dimensions [1,2]. In this process, the liquid (or solution) is charged by the nozzle which is connected to a high-voltage power supply. The electrostatic force incurred by these charges surmounts the surface tension of the solution and splits the solution jet into very small droplets. Tiny droplets with charges resist one another and droplet consolidation can be minimized, which in turn produces droplets with a uniform size distribution [3].

The electrospraying, with charged droplets, possesses advantages over conventional mechanical spraying systems. For a droplet produced from a solution, the remaining substance crystallizes and forms a solid particle. Electrosprayed droplets have size smaller than those obtained from conventional mechanical atomizers, and can be down to tens of nanometers. The size composition of the droplets is generally narrow with a low standard deviation. Electrospraying of biodegradable polymers, such as poly-lactide-co-glycolide (PLGA), polycaprolactone (PCL) etc. has found its biggest applications in biomedical engineering [4-7] and drug delivery [8-14]. Despite all the advantages associated 
with electrospraying, nevertheless, the optimization of the spraying procedure has been based on a time-consuming trial-and-error process.

In past three decades, PLGA has been one of the most attractive polymeric materials used to manufacture devices for drug delivery and tissue engineering applications [15]. PLGA is biocompatible and biodegradable, possesses a wide range of erosion times, has tunable mechanical strengths, and most importantly, is a FDA approved polymer. Crystalline PGA, when co-polymerized with PLA, reduces the degree of crystallinity of PLGA, and as a result, increases the rate of hydration and hydrolysis. As a rule, a higher content of PGA leads to quicker rates of degradation, with an exception of 50:50 ratio of PLA/PGA, which exhibits the fastest degradation. A ratio of 50:50 PLGAs have been extensively studied for the development of devices for controlled delivery of small molecule drugs, proteins and other macromolecules in commercial use and in research [16].

An empirical study, based on a fractional orthogonal array design, was completed to investigate the effect of different processing factors on the size distribution of electrosprayed particles. 50:50 PLGA materials were selected. Experiments were carried out on an electrospraying setup consisting of a syringe and needle, a ground electrode, and a high voltage supply. After electrospraying, the particle size and morphology were characterized by a scanning electron microscope. A fractional orthogonal was designed based on a design matrix proposed by Dr. Genichi Taguchi [17]. It allows one to examine a selected subset of combinations of multiple factors at multiple levels, but with less experimental test trials for optimization of the electrospraying process.

\section{Materials and Methods}

\subsection{Polymer Materials}

The polymeric material, adopted in this study, was a commercially available poly (d,1)-lactide-co-glycolide (PLGA) (Resomer RG 503, Boehringer, Germany) that has a LA:GA ratio of 50:50 and a molecular weight of 33,000 Da.

\subsection{Experimental Setup}

The electrospraying setup utilized in this study consisted of a syringe pump, a syringe and needle, a ground collection disk, and a high voltage supply. The needle was linked to the high voltage supply (You-Shang Tech. Corp., Kaohsiung, Taiwan), which was able to produce positive DC voltages and current up to $35 \mathrm{kV}$, and $4.16 \mathrm{~mA} / 125 \mathrm{~W}$, respectively. For the electrospraying of PLGA particles, PLGA was first dissolved in the solvent at a preset concentration. The dissolved liquid was then transported by a syringe pump (Kd Scientific Inc., Holliston, MA, USA), through a polytetrafluoroethylene (PTFE) tube, to the needle at a predetermined volumetric flow rate. All electrospraying experiments were carried out under a temperature of $25^{\circ} \mathrm{C}$ and a relative humidity of $60 \%$. Figure 1 shows the setup employed in this study.

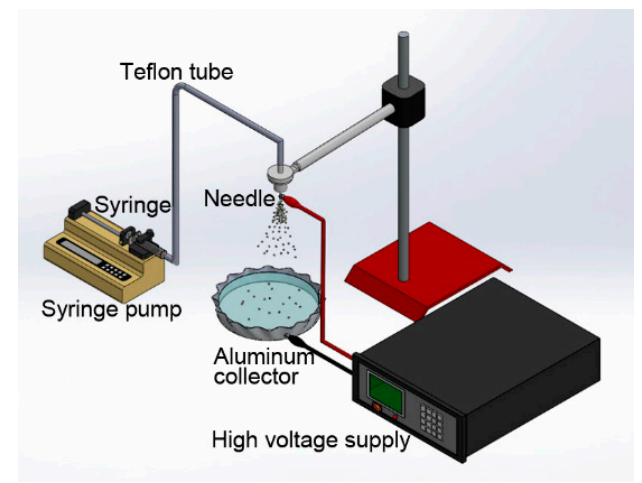

Figure 1. Schematically the electrospraying setup, which consisted of a syringe pump, a syringe and a needle, a Teflon tube, a ground collection disk, and a high voltage supply. 


\subsection{Empirical Factors and Orthogonal Design}

We selected six factors for analysis, including PLGA concentration in the solution, volumetric flow rate of the solution, travel distance between the needle tip and the ground collection disk, positive voltage applied to polymer solutions, size of the needle, and type of solvent used. The concentration of PLGA in the solution was 3, 5, or $7 \%(w / v)$. The flow rate of the solution was $0.6,0.8$, or $1.0 \mathrm{~mL} / \mathrm{h}$. The travel distance was set at 10,13 , or $15 \mathrm{~cm}$. The voltage was maintained at 11 ,

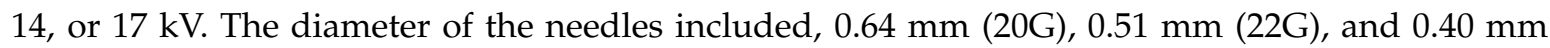
(24G). Meanwhile, three types of solvents were employed, namely hexafluoro-2-propanol (HFIP), dichloromethane (DCM), and trichloromethane (TCM). Table 1 shows the selected factors and factor levels.

Table 1. Factors and factor levels selected in the main experiment.

\begin{tabular}{cccc}
\hline Factors & Level 1 & Level 2 & Level 3 \\
\hline A: Polymer concentration $(\%, w / v)$ & 3 & 5 & 7 \\
B: Flow rate $(\mathrm{mL} / \mathrm{h})$ & 0.6 & 0.8 & 1.0 \\
C: Travel distance $(\mathrm{cm})$ & 10 & 13 & 15 \\
D: Voltage $(\mathrm{kV})$ & 11 & 14 & 17 \\
E: Size of needle $(\mathrm{mm})$ & 0.64 & 0.51 & 0.40 \\
F: Solvent & DCM & HFIP & TCM \\
\hline
\end{tabular}

DCM: Dichloromethane; HFIP: Hexafluoro-2-propanol; TCM: Trichloromethane.

After electrospraying, the morphology of electrosprayed particles was inspected under a field emission scanning electron microscope (FESEM; JEOL Model JSM-7500F, Tokyo, Japan). The particle sizes were determined by manually evaluating SEM photos of 30 randomly selected particles for each test run $(n=3)$. We adopted the L18 orthogonal array design (Table 2 ) for analysis. The array stipulates the way of conducting the minimal number of experiments, which could provide the full information of all the factors that influence the performance parameter [17]. Since the electrospraying of tiny-sized particles is desired in this study, the optimum processing condition could be acquired by integrating all the factor levels that possess the smallest particle sizes.

Table 2. $L^{\prime} 18\left(3^{6}\right)$ orthogonal array used in the main experiment.

\begin{tabular}{ccccccc}
\hline Run & $\begin{array}{c}\text { A: Polymer } \\
\text { Concentration }(\%, w / v)\end{array}$ & $\begin{array}{c}\text { B: Flow rate } \\
(\mathbf{m L} / \mathbf{h})\end{array}$ & $\begin{array}{c}\text { C: Travel } \\
\text { Distance }(\mathbf{c m})\end{array}$ & $\begin{array}{c}\text { D: Voltage } \\
(\mathbf{k V})\end{array}$ & $\begin{array}{c}\text { E: Size of } \\
\text { Needle }(\mathbf{m m})\end{array}$ & F: Solvent \\
\hline 1 & $3 \%$ & 0.6 & 10 & 11 & 0.64 & DCM \\
2 & $3 \%$ & 0.8 & 13 & 14 & 0.51 & HFIP \\
3 & $3 \%$ & 1 & 15 & 17 & 0.40 & TCM \\
4 & $5 \%$ & 0.6 & 10 & 14 & 0.51 & TCM \\
5 & $5 \%$ & 0.8 & 13 & 17 & 0.40 & DCM \\
6 & $5 \%$ & 1 & 15 & 11 & 0.64 & HFIP \\
7 & $7 \%$ & 0.6 & 13 & 11 & 0.40 & HFIP \\
8 & $7 \%$ & 0.8 & 15 & 14 & 0.64 & TCM \\
9 & $7 \%$ & 1 & 10 & 17 & 0.51 & DCM \\
10 & $3 \%$ & 0.6 & 15 & 17 & 0.51 & HFIP \\
11 & $3 \%$ & 0.8 & 10 & 11 & 0.40 & TCM \\
12 & $3 \%$ & 1 & 13 & 14 & 0.64 & DCM \\
13 & $5 \%$ & 0.6 & 13 & 17 & 0.64 & TCM \\
14 & $5 \%$ & 0.8 & 15 & 11 & 0.51 & DCM \\
15 & $5 \%$ & 1 & 10 & 14 & 0.40 & HFIP \\
16 & $7 \%$ & 0.6 & 15 & 14 & 0.40 & DCM \\
17 & $7 \%$ & 0.8 & 10 & 17 & 0.64 & HFIP \\
18 & $7 \%$ & 1 & 13 & 11 & 0.51 & TCM \\
\hline
\end{tabular}

DCM: Dichloromethane; HFIP: Hexafluoro-2-propanol; TCM: Trichloromethane. 


\section{Results and Discussions}

Poly(lactic-co-glycolic acid) (PLGA) has been one of the most successfully developed and extensively researched synthetic biomaterials $[15,16]$. It has also been widely adopted to produce polymeric microparticles, mainly due to their attractive properties, including biodegradability and biocompatibility, and sustained target delivery to specific organs or cells.

Experiments were completed on a lab-scale electrospraying setup. Figure 2 displays the SEM images and the size distribution of electrosprayed microspheres for the 18 test experiments, while Table 3 lists the properties of these particles. The property acquired from each experimental trial is then evaluated statistically.
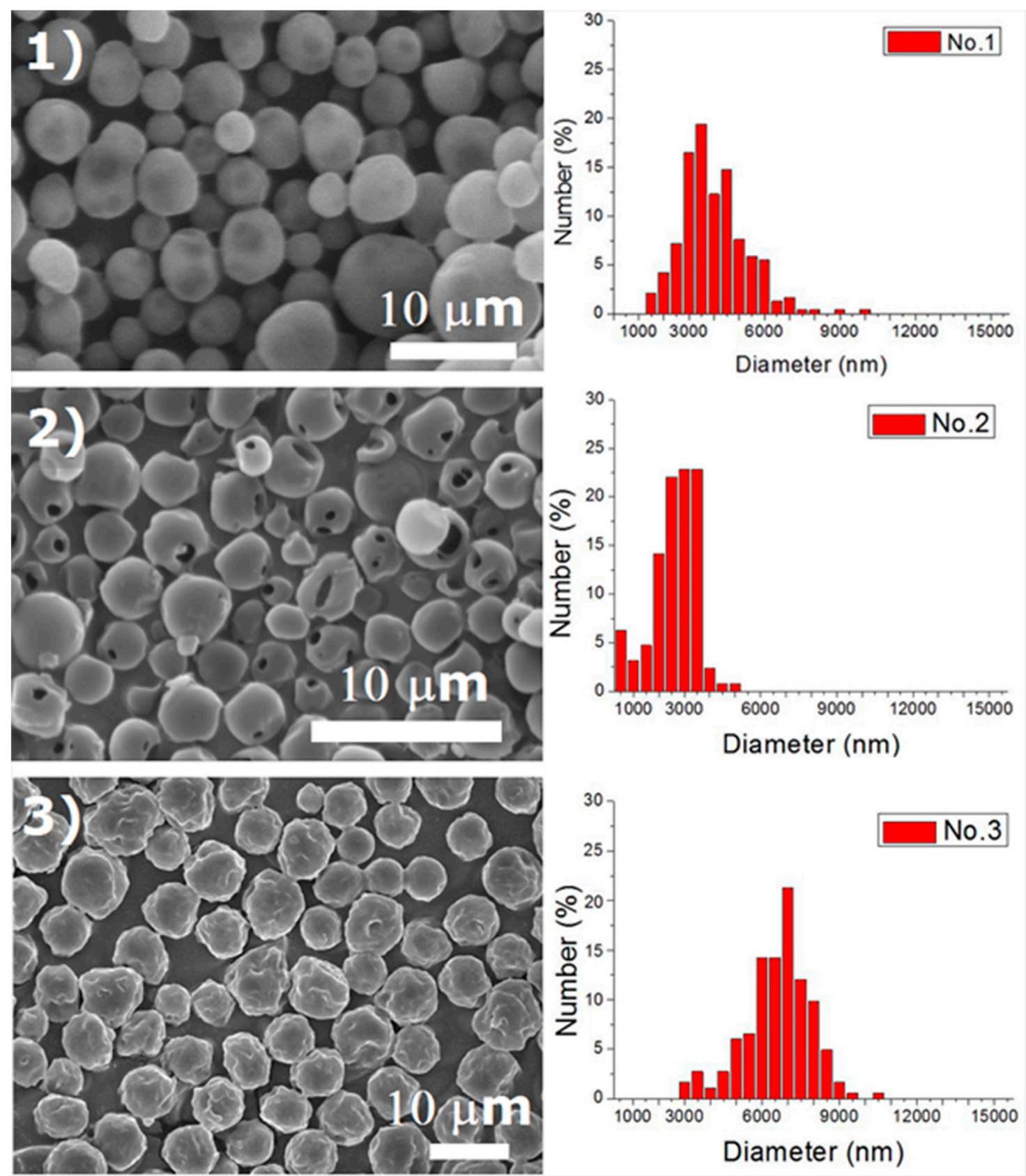

Figure 2. Cont. 

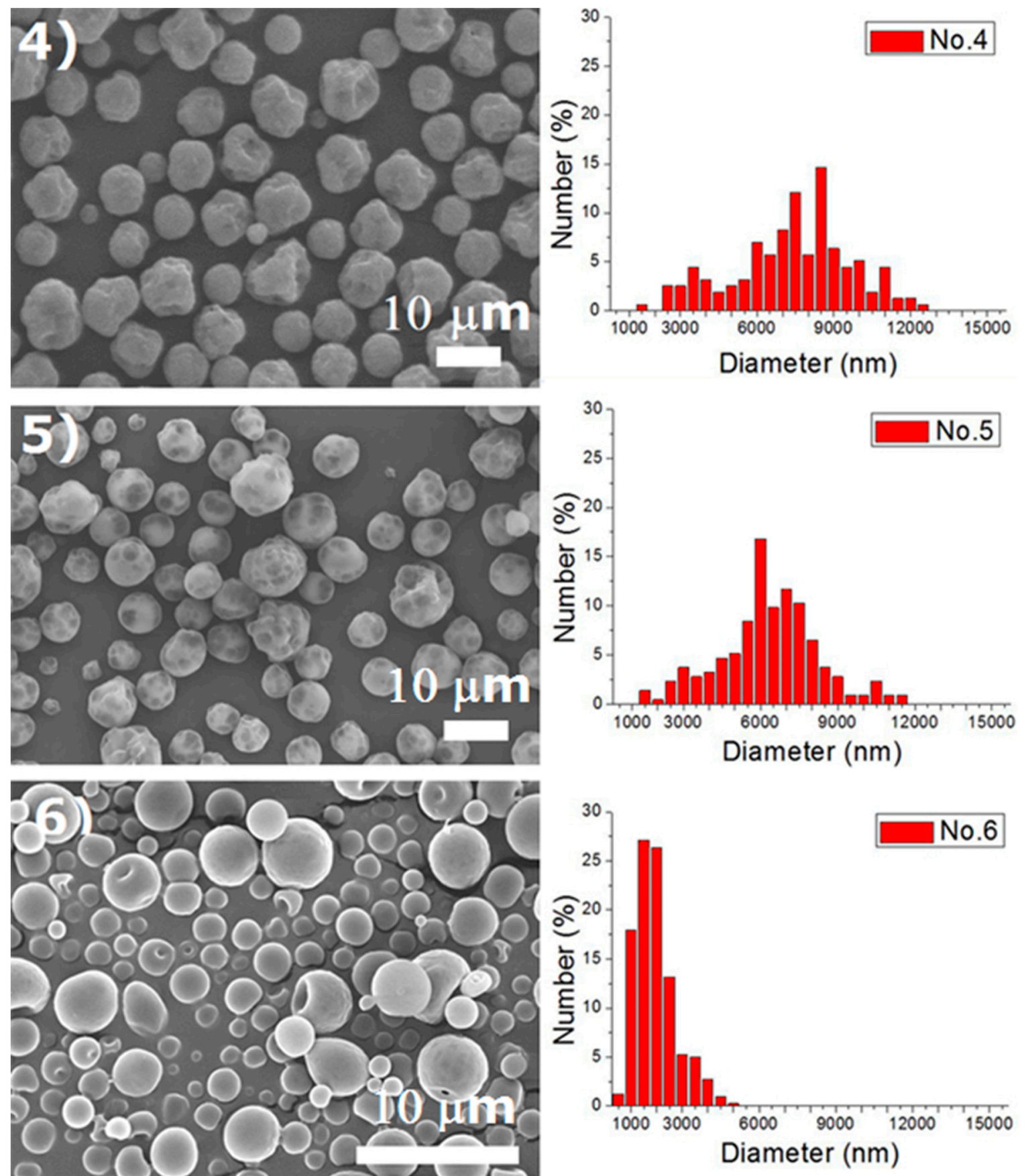

Figure 2. Cont. 

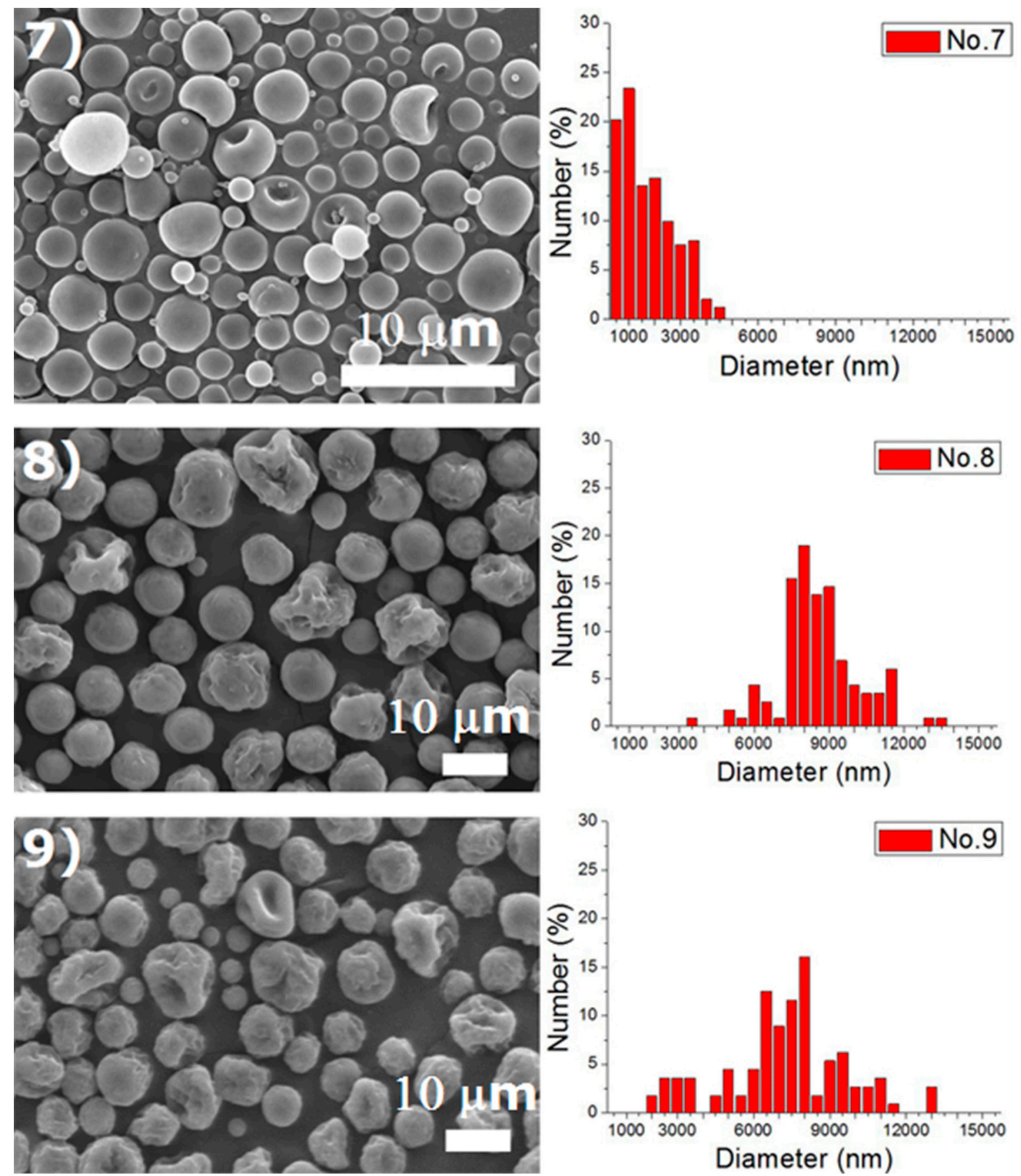

Figure 2. Cont. 

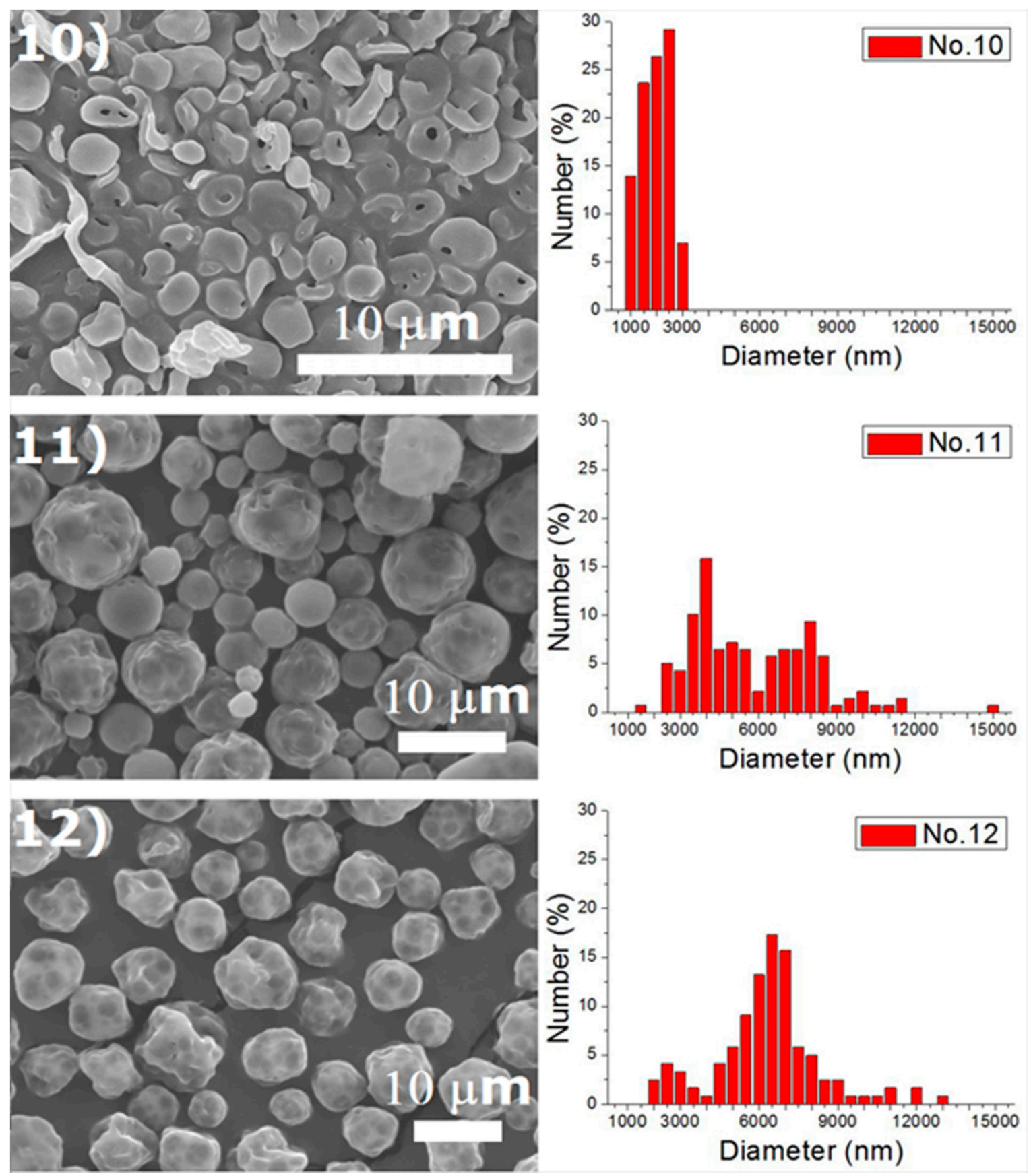

Figure 2. Cont. 

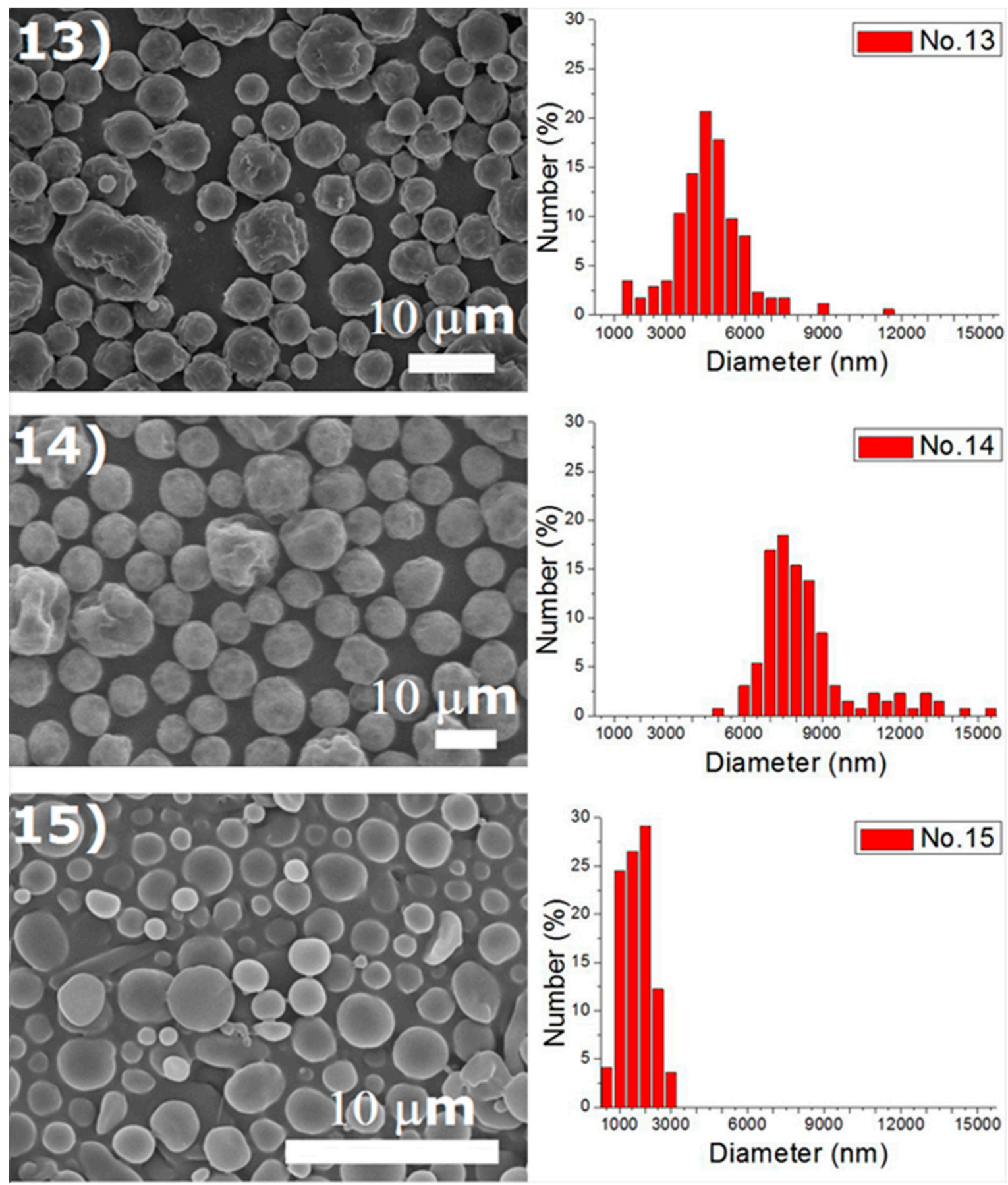

Figure 2. Cont. 

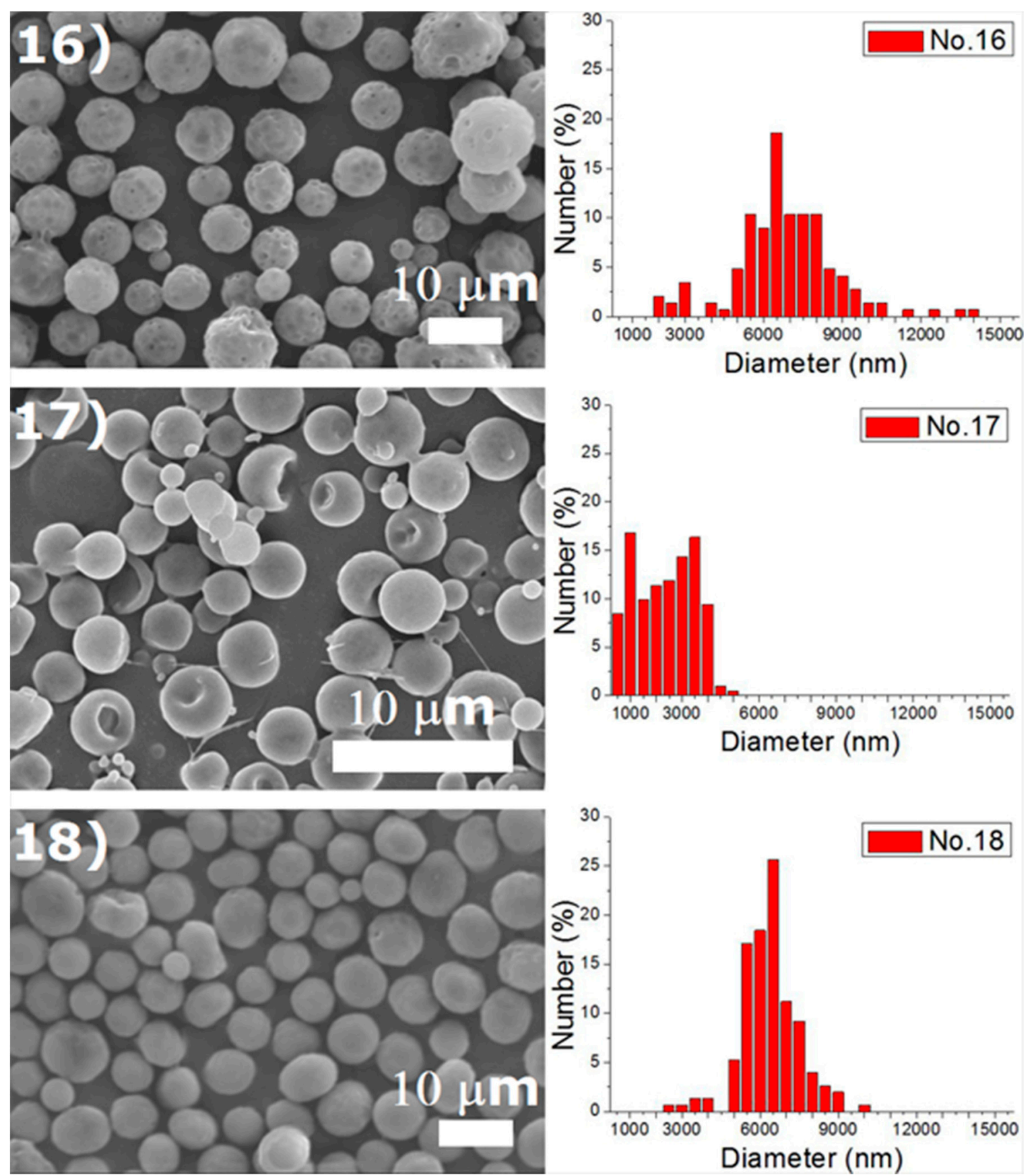

Figure 2. SEM photos and particle size distribution of electrosprayed microspheres (Test runs 1-18).

\subsection{Optimization of Processing Parameters}

The signal-to-noise ratio $(S / N)$ is the statistical value, measuring how the response varies relative to the nominal or target value under different noise conditions [17]. Higher values of the signal-to-noise ratio $(S / N)$ identify control factor settings that minimize the effects of the noise factors. Maximizing the $S / N$ ratio results in minimized properties that are sensitive to noise. Since the reduction of particle size is the object of this work, the expression depicting the smaller-the-better feature was adopted for the analysis:

$$
\frac{S}{N}=-10 \log _{10}\left[\left(\frac{1}{n}\right) \sum_{i=1}^{n}\left(\bar{y}^{2}+S_{n}^{2}\right)\right]
$$


where $\bar{y}$, and $S_{n}$ are the average value, and deviation of the calculated particle size, respectively, and $n$ represents the number of specimen for each test run. The factor levels with the greatest $S / N$ ratios will minimize the noises sensitivity and lead to the optimum levels.

The change of particle size, due to various factors, was evaluated based on the technique proposed by Taguchi [17]. Calculated $S / N$ ratios for the sizes of electrosprayed PLGA particles are displayed in Figure 3. According to the figure, the optimized factor levels, which lead to the minimum particle size, were estimated to be $A 1 / B 1 / C 1 / D 1 / E 1 / F 2$. These optimum factor levels stand for a polymer concentration of $3 \%$, a flow rate of $0.6 \mathrm{~mL} / \mathrm{h}$, a travel distance of $10 \mathrm{~cm}$, a voltage of $11 \mathrm{kV}$, a needle size of $0.64 \mathrm{~mm}$, and HFIP solvent.

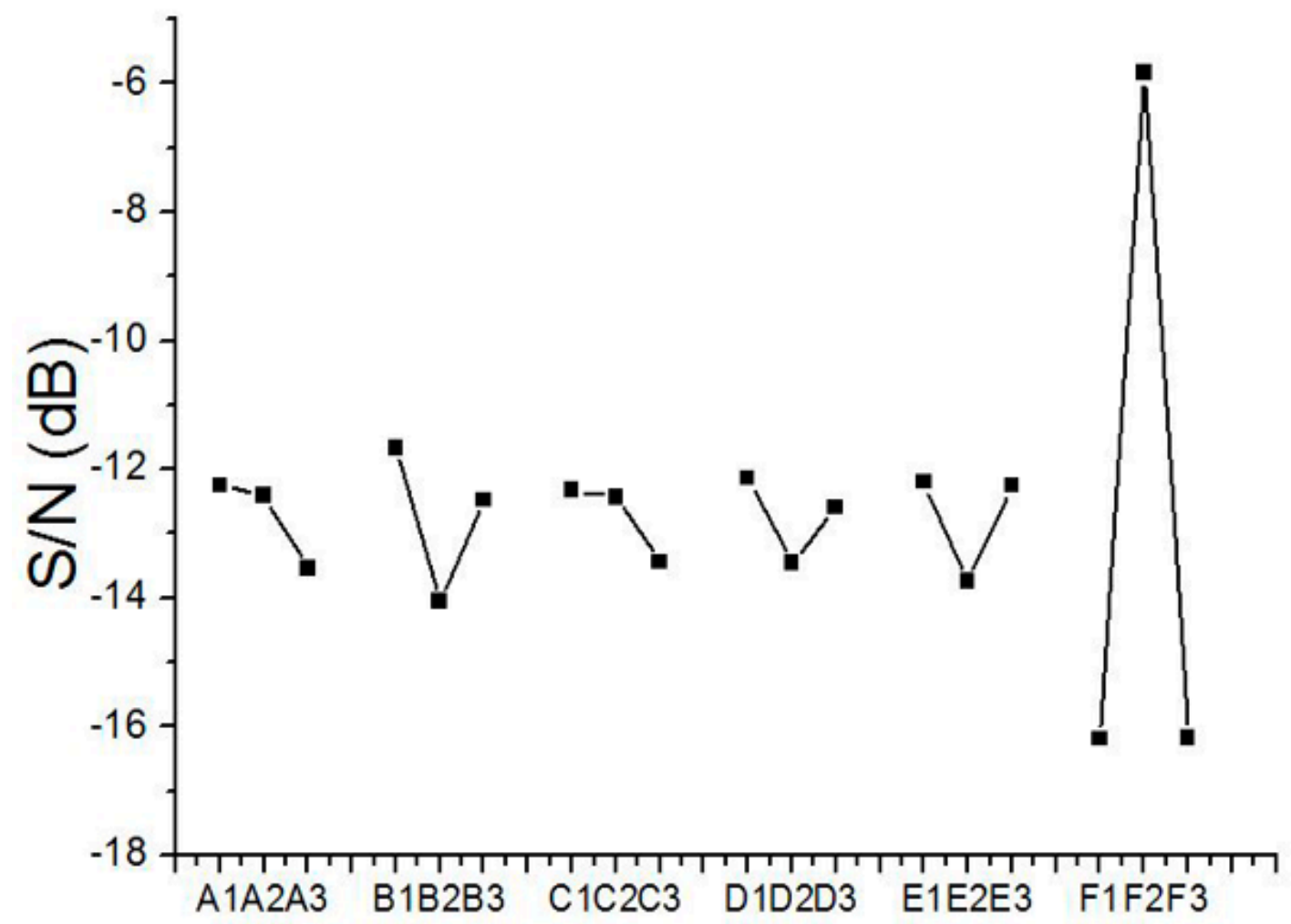

Figure 3. Variation of the $S / N$ ratio with factor levels for electrosprayed poly (d,l)-lactide-co-glycolide (PLGA) particles.

Since the optimum integration of factor levels was not contained in the L18 array experiment, an alternative path was engaged to estimate the response of the particle size to the optimum factor levels. Presuming there was no interaction among the chosen factors, the estimated $S / N$ ratio for the optimum levels, $\eta_{A 1 / B 1 / C 1 / D 1 / E 1 / F 2}$, is

$$
\begin{gathered}
\eta_{A 1 B 1 C 1 D 1 E 1 F 2}=\eta_{m}+\left(\eta_{A 1}-\eta_{m}\right)+\left(\eta_{B 1}-\eta_{m}\right)+\left(\eta_{C 1}-\eta_{m}\right)+\left(\eta_{D 1}-\eta_{m}\right)+\left(\eta_{E 1}-\eta_{m}\right)+\left(\eta_{F 2}-\eta_{m}\right) \\
=\eta_{A 1}+\eta_{B 1}+\eta_{C 1}+\eta_{D 1}+\eta_{E 1}+\eta_{F 2}-5 \eta_{m}
\end{gathered}
$$

where $\eta_{m}$ is the mean $S / N$ ratio for the experimental tests in Table 3 , and $\eta_{F N}$ is the $S / N$ ratio for factor $\mathrm{F}$ and level $\mathrm{N}$. On the basis of this equation, the estimated $S / N$ ratio of electrosprayed particle sizes for the optimum levels, $A 1 / B 1 / C 1 / D 1 / E 1 / F 2$, was $-2.75 \mathrm{~dB}$. This estimated value was surely higher than those attained in the 18 test trials in Table 3.

A verification test was completed in line with the optimum factor levels. The optimized levels for electrosprayed particles are $A 1 / B 1 / C 1 / D 1 / E 1 / F 2$. The particle size thus acquired was $1.43 \pm 0.93 \mu \mathrm{m}$ (as displayed in Figure 4). Furthermore, the $S / N$ ratio for this verification experiment was $-4.67 \mathrm{~dB}$. Although this value is lower than the estimated value of $-2.75 \mathrm{~dB}$, and the one prepared using 
processing condition $15(-3.63 \mathrm{~dB})$, it is higher than those achieved by other processing conditions in the 18 test trials in Table 3. Furthermore, when compared with the size distribution of particles produced by condition 15 (Figure 2), microspheres prepared using the optimum condition showed a greater percentage $(47.72 \%)$ of particles with a size less than $1000 \mathrm{~nm}$. Consequently, by employing the optimum factor levels, the particle sizes of electrosprayed microspheres were adequately minimized.

Table 3. Tabulation of size distribution for electrosprayed particles in the main experiment.

\begin{tabular}{ccc}
\hline Run & Particle Size $(\mu \mathrm{m})$ & S/N Ratio \\
\hline 1 & $3.88 \pm 1.30$ & -12.25 \\
2 & $2.38 \pm 0.87$ & -8.08 \\
3 & $6.36 \pm 1.30$ & -16.24 \\
4 & $7.15 \pm 2.29$ & -17.51 \\
5 & $6.09 \pm 1.93$ & -16.11 \\
6 & $1.71 \pm 0.79$ & -5.52 \\
7 & $1.50 \pm 1.00$ & -5.14 \\
8 & $8.34 \pm 1.62$ & -18.58 \\
9 & $7.01 \pm 2.37$ & -17.38 \\
10 & $1.71 \pm 0.56$ & -5.11 \\
11 & $5.56 \pm 2.33$ & -15.61 \\
12 & $6.08 \pm 2.02$ & -16.14 \\
13 & $4.36 \pm 1.36$ & -13.20 \\
14 & $8.15 \pm 1.83$ & -18.44 \\
15 & $1.40 \pm 0.57$ & -3.63 \\
16 & $6.59 \pm 1.96$ & -16.75 \\
17 & $2.09 \pm 1.10$ & -7.49 \\
18 & $6.12 \pm 1.10$ & -15.87 \\
\hline
\end{tabular}
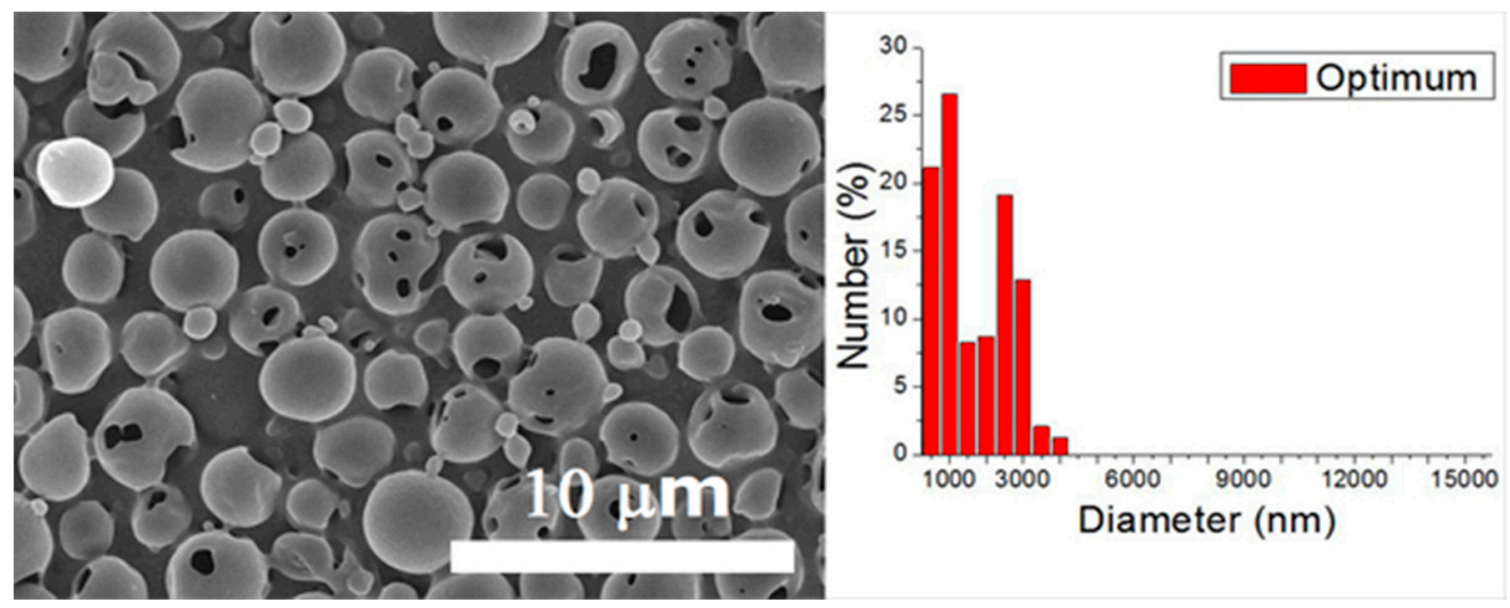

Figure 4. SEM photo and size distribution of particles electrosprayed using the optimum processing condition.

\subsection{Significance of Factors}

A standard analysis of variance (ANOVA) was completed in line with the technique proposed by Taguchi [17]. Table 4 lists the computed results for electrosprayed PLGA particles. The variance ratio, represented by $\mathrm{F}$ in Table 4, is calculated by dividing the factor mean square by the error mean square. A larger value of $\mathrm{F}$ indicates that the significance of that factor is greater compared with the error variance. The importance of each factor on the particle size of electrosprayed PLGA microspheres can be determined by the values of $\mathrm{F}$ in Table 4 . On the basis of Table 4, the relative importance of each factor on the particle size of electrosprayed PLGA microspheres was, in decreasing order, solvent type $(F=49.02)$, flow rate $(F=2.76)$, travel distance $(F=2.46)$, size of needle $(F=2.10)$, polymer concentration 
$(\mathrm{F}=1.62)$, and applied voltage $(\mathrm{F}=1.49)$, respectively. For the factors chosen in this analysis, the solvent used was found to be the key factor affecting the particle size of electrosprayed microspheres.

Table 4. Standard analysis of variance (ANOVA) table for the size distribution of electrosprayed particles.

\begin{tabular}{ccccc}
\hline Factor & Degree of Freedom & Sum of Squares & Mean Square & F \\
\hline A-Polymer concentration $(\%)$ & 2 & 8.08 & 4.04 & 1.62 \\
B-Flow rate $(\mathrm{mL} / \mathrm{h})$ & 2 & 13.75 & 6.87 & 2.76 \\
C-Travel distance $(\mathrm{cm})$ & 2 & 12.24 & 6.12 & 2.46 \\
D-Voltage $(\mathrm{kV})$ & 2 & 7.43 & 3.71 & 1.49 \\
E-Needle size $(\mathrm{mm})$ & 2 & 10.48 & 5.24 & 2.10 \\
F-Solvent type & 2 & 244.07 & 122.03 & 49.02 \\
Error & 36 & 89.61 & 2.48 & \\
\hline
\end{tabular}

The statistical results in Figure 3 (factor level F2) suggest that one should use hexafluoro-2-propanol (HFIP) as the solvent to acquire the optimized microparticles. HFIP is a fluorinated alcohol frequently used as solvent for polymer systems. HFIP shows strong hydrogen bonding properties causing it to better dissolve a number of molecules with suitable receptive sites such as oxygen atoms, double bonds or amine groups (Sigma-Aldrich, St. Louis, MO, USA) [18]. It is easier for the HFIP dissolved PLGA solution to be stretched and break into small droplets by the external electrical forces during the process. Electrosprayed microparticles thus showed the smallest diameter distribution.

DCM was found to electrospray microspheres with a fairly spherical geometry, while TCM electrosprayed particles with relatively rougher surfaces. Surface tension is well known to be responsible for the spherical shape of liquid droplets. TCM has a higher polarity than DCM, which makes itself a good solvent for a wide range of reactions [18]. The polar force of TCM may counteract the solution's surface tension and result in a rough surface of the droplets and the relevant solid particles. Relatively, DCM has a lower polarity and thus produces microparticles with a more spherical surface. On the other hand, hollowed particles could be observed in particles prepared using HFIP, such as those prepared by conditions $1,6,7,10$ in Table 2 . The only exceptions are the ones produced using a higher flow rate (conditions 6 and 15), namely $1.0 \mathrm{~mL} / \mathrm{h}$. This might be due to the fact that HFIP evaporates faster than DCM and TCM, and may leave a hollowed core after evaporation. However, when the flow rate is high, the excessive polymeric material may be able to compensate the hollowed material at the core. Electrosprayed particles thus exhibited a less hollowed geometry.

The data in the main experiment (factor level B1 in Figure 3) suggest that the PLGA materials electrosprayed with the least flow rate resulted in microparticles of smallest diameter distribution. It has been reported that there is a positive correlation between PLGA solution flow rate and particle size, so that, as the flow rate decreases, the size of electrosprayed microparticles decreases also [19]. The smallest flow rate $(0.6 \mathrm{~mL} / \mathrm{h})$ employed in the experiments thus produced particles with the smallest size distribution.

The experimental results in factor level B1 of Figure 3, suggested that one can obtain microparticles of the smallest diameter by utilizing the least travel distance. During the electrospraying process, the polymeric solution ejected from the nozzle was stretched and broken into tiny droplets by the electrical fields. With a shorter distance, the electrical force applied to the droplets is higher. It is thus easier for the droplets to break into tiny particles.

The empirical data in Figure 3 (factor level A1) suggested that one should use the lowest PLGA concentration $(3 \%)$ to spray microspheres of smallest diameters. This is due to the fact that to produce smaller droplets and uniform microparticles and, it is necessary to limit chain entanglements [20,21]. During the electrospraying process, solvent evaporation and polymer chain diffusion occur during the travel of solution droplets to the collector, and chain entanglement is responsible for the final morphology of particles [22]. It has been reported that, to prepare particles by electrospraying technique, the best polymeric solution is a semi-dilute moderately entangled regime, which takes place at the critical entanglement concentration (Ce) [23]. This stage is the crossover point from 
the semi-dilute unentangled regime to the semi-dilute moderately entangled regime. Within this range, there is enough entanglement, and dense, solid, and reproducible particles can be prepared. Faramarzi et al. [24] studied the effect of solution on the morphology and size of electrosprayed PLGA particles, and proposed that polymeric concentration of $2 \sim 4 \mathrm{wt}$. $\%$ falls into the range of a semi-dilute, moderately entangled regime and chain entanglement can easily occur for the electrospraying of microparticles. Nevertheless, as the concentration increased to 6-8\%, the semi-dilute highly entangled regime was formed and a variety of tailed particles and fibers started to appear along with the particles. The optimum PLGA concentration obtained in this study was 3\%, which matches well with the results of the literature [24]. A polymeric concentration that is too high in the solution leads to major chain entanglements in the polymeric materials. The solution thus possesses higher strengths to resist the electric field from breaking into tiny drops. Electrosprayed microspheres thus possess a greater size distribution, and may sometime exhibit tailed geometries.

Finally, despite the applied voltage required component in achieving sustainable solution-jet mode during electrospraying, it showed a relatively insignificant influence on the particle size distribution of electrosprayed PLGA microspheres, as suggested by the result in Table 4.

This study has successfully employed the Taguchi experimental design to minimize the particle size of electrosprayed PLGA microspheres. The same scheme can also be applied to optimize the electrospraying of other materials. Furthermore, if a specific desired particle size is desired, the "nominal-the-best" formulation [17] can also be adopted to optimize the desired size distribution easily. This further demonstrates the feasibility of the experimental design in manufacturing microspheres for various applications.

\section{Conclusions}

The following can be obtained on the basis of the experimental results presented:

1. Orthogonal array design based on the Taguchi technique was adequate in minimizing the particle size of electrosprayed microspheres by optimizing the processing factors, despite the interaction among the chosen factors was ignored in the evaluation.

2. For the factors selected in the experiments, the type of solvent used was discovered to be the principal factor affecting the particle size of electrosprayed microspheres. HFIP electrosprayed microparticles had the smallest diameter. However, hollowed particles could be seen among these microspheres.

3. DCM was found to electrospray microspheres with a fairly spherical geometry, while TCM electrosprayed particles with relatively rougher surfaces.

4. Particle size of sprayed microspheres decreases somewhat with the polymer concentration and the travel distance.

Author Contributions: Conceptualization was handled by M.-Y.H. and S.-J.L., funding acquisition was handled by S.-J.L., the investigation was handled by C.-H.F. and Y.-W.L., the manuscript was written by S.-J.L. and M.-Y.H., and supervision of the research was handled by S.-J.L.

Funding: The authors would like to thank the Ministry of Science and Technology, Taiwan (Contract No. 107-2221-E-182-017) and the Chang Gung Memorial Hospital (Contract No. CMRPD2H0032) for financially supporting this study.

Conflicts of Interest: The authors declare that there is no conflict of interest regarding the publication of this paper.

\section{References}

1. Gaskell, S.M. Electrospray: Principles and Practice. J. Mass Spectrom. 1997, 32, 677-688. [CrossRef]

2. Jaworek, A. Micro- and nanoparticle production by electrospraying. Powder Technol. 2007, 176, 18-35. [CrossRef]

3. Boda, S.K.; Li, X.; Xie, J. Electrospraying an enabling technology for pharmaceutical and biomedical applications: A review. J. Aerosol Sci. 2018. [CrossRef] 
4. Bock, N.; Woodruff, M.A.; Hutmacher, D.W.; Dargaville, T.R. Electrospraying, a reproducible method for production of polymeric microspheres for biomedical applications. Polymers 2011, 3, 131-149. [CrossRef]

5. Enayati, M.; Chang, M.-W.; Bragman, F.; Edirisinghe, M.; Stride, E. Electrohydrodynamic preparation of particles, capsules and bubbles for biomedical engineering applications. Colloids Surf. A 2011, 382, 154-164. [CrossRef]

6. Danhier, F.; Ansorena, E.; Silva, J.M.; Coco, R.; Breton, A.L.; Preat, V. PLGA-based nanoparticles: An overview of biomedical applications. J. Control Release 2012, 161, 505-522. [CrossRef]

7. Nguyen, D.N.; Clasen, C.; Van den Mooter, G. Pharmaceutical applications of electrospraying. J. Pharm. Sci. 2016, 105, 2601-2620. [CrossRef]

8. Sridhar, R.; Ramakrishna, S. Electrosprayed nanoparticles for drug delivery and pharmaceutical applications. Biomatter 2013, 3, e24281. [CrossRef]

9. Peltonen, L.; Valo, H.; Kolakovic, R.; Laaksonen, T.; Hirvonen, J. Electrospraying, spray drying and related techniques for production and formulation of drug nanoparticles. Expert Opin. Drug Deliv. 2010, 7, 705-719. [CrossRef]

10. Hao, S.; Wang, Y.; Wang, B.; Deng, J.; Liu, X.; Liu, J. Rapid preparation of pH-sensitive polymeric nanoparticle with high loading capacity using electrospray for oral drug delivery. Mater. Sci. Eng. C 2013, 33, 4562-4567. [CrossRef]

11. Prabhakaran, M.P.; Zamani, M.; Felice, B.; Ramakrishna, S. Electrospraying technique for the fabrication of metronidazole contained PLGA particles and their release profile. Mater. Sci. Eng. C 2015, 56, 66-73. [CrossRef] [PubMed]

12. Hao, S.; Wang, Y.; Wang, B.; Deng, J.; Zhu, L.; Cao, Y. Formulation of porous poly(lactic-co-glycolic acid) microparticles by electrospray deposition method for controlled drug release. Mater. Sci. Eng. C 2014, 39, 113-119. [CrossRef] [PubMed]

13. Songsurang, K.; Praphairaksit, N.; Siraleartmukul, K.; Muangsin, N. Electrospray fabrication of doxorubicin-chitosan-tripolyphosphate nanoparticles for delivery of doxorubicin. Arch. Pharm. Res. 2011, 34, 583-592. [CrossRef] [PubMed]

14. Malik, S.A.; Ng, W.H.; Bowen, J.; Tang, J.; Gomez, A.; Kenyon, A.J.; Richard, M.; Day, R.M. Electrospray synthesis and properties of hierarchically structured PLGA TIPS microspheres for use as controlled release technologies. J. Colloid Interface Sci. 2016, 467, 220-229. [CrossRef]

15. Makadia, H.K.; Siegel, S.J. Poly lactic-co-glycolic acid (PLGA) as biodegradable controlled drug delivery carrier. Polymers 2011, 3, 1377-1397. [CrossRef]

16. Sah, E.; Sah, H. Recent trends in preparation of poly(lactide-co-glycolide) nanoparticles by mixing polymeric organic solution with antisolvent. J. Nanomater. 2015, 16, e794601. [CrossRef]

17. Roy, R.K. A Primer on the Taguchi Method; Society of Manufacturing Engineering: New York, NY, USA, 1990.

18. Reichardt, C.; Welton, T. Solvents and Solvent Effects in Organic Chemistry, 4th ed.; Wiley-VCH Verlag: Weinheim, Germany, 2011.

19. Mayol, L.; Borzacchiello, A.; Guarino, V.; Serri, C.; Biondi, M.; Ambrosio, L. Design of electrosprayed non-spherical poly (L-lactide-co-glicoide) microdevices for sustained drug delivery. J. Mater. Sci. Mater. Med. 2014, 25, 383-390. [CrossRef]

20. Bock, N.; Dargaville, T.R.; Woodruff, M.A. Electrospraying of polymers with therapeutic molecules: State of the art. Prog. Polym. Sci. 2012, 37, 1510-1551. [CrossRef]

21. Liu, S.J.; Kau, Y.C.; Chou, C.Y.; Chen, J.K.; Wu, R.C.; Yeh, W.L. Electrospun PLGA/collagen nanofibrous membrane as early-stage wound dressing. J. Membr. Sci. 2010, 355, 53-59. [CrossRef]

22. Wu, Y.; Clark, R.L. Controllable porous polymer particles generated by electrospraying. J. Colloid Interface Sci. 2007, 310, 529-535. [CrossRef] [PubMed]

23. Costa, L.M.M.; Bretas, R.E.S.; Gergorio, R., Jr. Effect of solution concentration on the electrospray/electrospinning transition and on the crystalline phase of PVDF. Mater. Sci. Appl. 2010, 1, 247-252. [CrossRef]

24. Faramarzi, A.-R.; Barzin, J.; Mobedi, H. Effect of solution and apparatus parameters on the morphology and size of electrosprayed PLGA microparticles. Fiber Polym. 2016, 117, 1806-1819. [CrossRef]

(C) 2019 by the authors. Licensee MDPI, Basel, Switzerland. This article is an open access article distributed under the terms and conditions of the Creative Commons Attribution (CC BY) license (http:/ / creativecommons.org/licenses/by/4.0/). 